CASE REPORT

\title{
Thrombus in Transit in Right Atrium with Ecograph Pulmonary Infarction in Patient with Massive Pulmonary Thromboembolism
}

\author{
Edroso Jarne Paloma Eugenia*, Arche Banzo Maria José, Pascual Bielsa Ana and Obón Azuara Blanca \\ Intensive Care Department, University Clinical Hospital, Spain \\ *Corresponding author: Edroso Jarne Paloma Eugenia, Intensive Care Department, University Clinical \\ Hospital, Zaragoza, Spain
}

We describe the case of a 61-year-old female who was admitted to our tertiary centre with 15-day of dyspnea and whitish expectoration. The patient had a history of deep vein thrombosis and pulmonary thromboembolism a year ago, anticoagulated until a few months ago with acenocumarol.

The patient was diagnosed with pulmonary thromboembolism by CT-scan and deep vein thrombosis in the proximal segment of the left femoral vein.

Anticoagulation was started with subcutaneous Heparin but this should be discontinued due to the patient developed severe hypotension, paleness and abdominal pain. Severe anemia was shown in blood

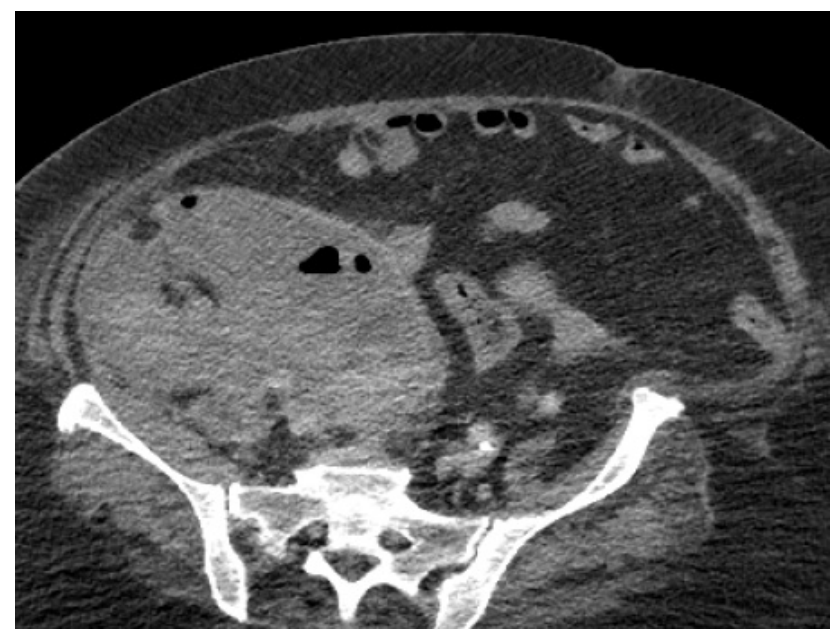

Figure 1: Large hematoma on psoas, oblique muscles and peritoneal cavity that displaces the right kidney in the anterior way, bladder and uterus. analysis. The patient was diagnosed with a large hematoma on psoas, oblique muscles and peritoneal cavity that displaces the right kidney in the anterior way, bladder and uterus (Figure 1). Embolization was performed with minicoils to L3 lumbar artery showing active bleeding.

The patient was admitted to the ICU. During admission to the Unit, an echocardiography was performed which showed linear mobile density in the right atrium

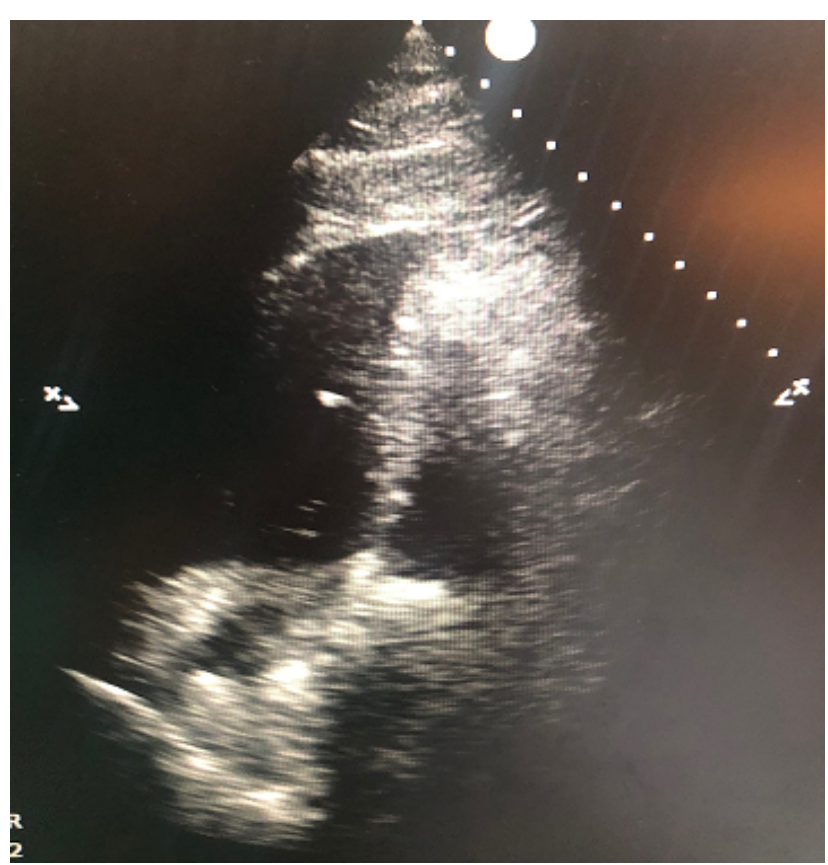

Figure 2: Linear mobile density in the right atrium that displaces atrial septum.

Citation: Eugenia EJP, José ABM, Ana PB, Blanca OA (2019) Thrombus in Transit in Right Atrium with Ecograph Pulmonary Infarction in Patient with Massive Pulmonary Thromboembolism. Int Arch Cardiovasc Dis 3:027. doi.org/10.23937/2643-3966/1710027

Accepted: December 14, 2019; Published: December 16, 2019

Copyright: (c) 2019 Eugenia EJP, et al. This is an open-access article distributed under the terms of the Creative Commons Attribution License, which permits unrestricted use, distribution, and reproduction in any medium, provided the original author and source are credited. 


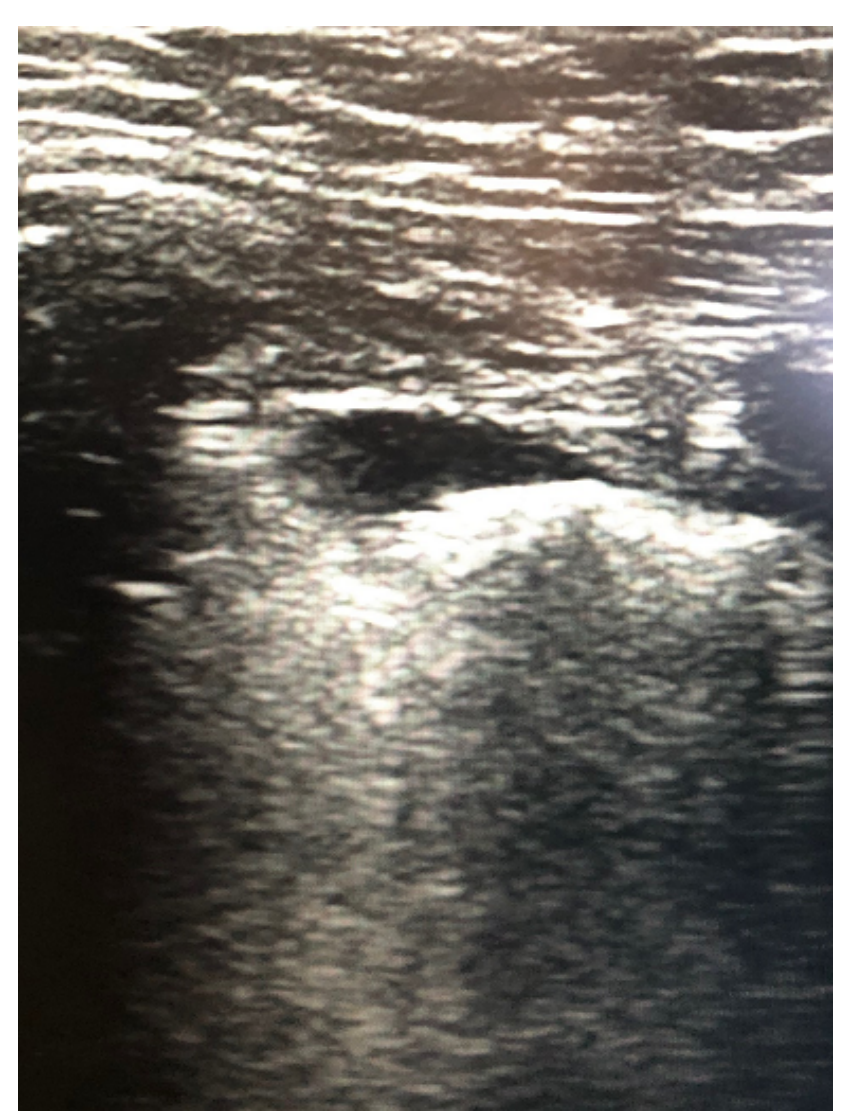

Figure 3: Image of pulmonary infarction.

that displaces atrial septum (Figure 2). In addition, a pulmonary ultrasound was performed showing an image of pulmonary infarction (Figure 3).

The case was discussed with the Heart Surgery Service who refused the intervention because of the high risk of new bleeding, as well as the evacuation of the

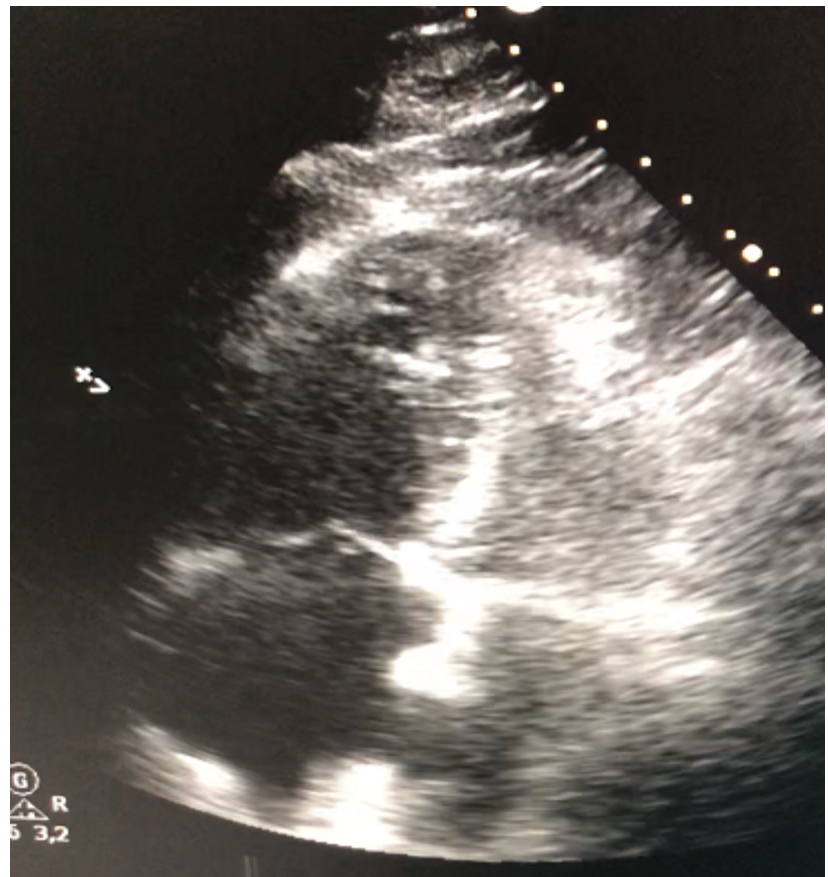

Figure 4: The exception of small remnant in roof of the right atrium.

hematoma by the General Surgery Service and the aspiration of intra-cardiac Clot by Interventional Radiology.

The patient remained stable hemodynamically and respiratoryly. After 48 hours without bleeding, being hemodynamically stable, it was decided to start anticoagulation with intravenous unfractionated heparin. 12 hours after the start, new echocardiography control was carried out showing the practical disappearance of the thrombus, with the exception of small remnant in roof of the right atrium (Figure 4).

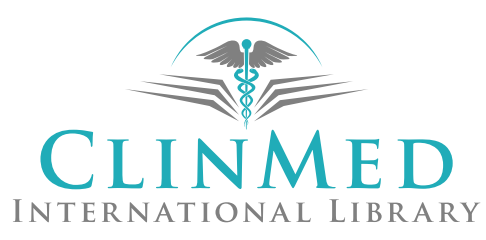

